Babelyuk V., Tserkovniuk R., Babelyuk N., Zukow X., Ruzhylo S., Dubkova G., Korolyshyn T., Hubyts'kyi V., Kikhtan V., Gozhenko A., \& Popovych I. The parameters of gas discharge visualization (biophotonics) correlated with parameters of acupuncture points, EEG, HRV and hormones. Journal of Education, Health and Sport. 2021;11(12):359-373. eISSN 2391-8306. DOI http://dx.doi.org/10.12775/JEHS.2021.11.12.030

https://apcz.umk.pl/JEHS/article/view/JEHS.2021.11.12.030

https://zenodo.org/record/5828274

The journal has had 40 points in Ministry of Education and Science of Poland parametric evaluation. Annex to the announcement of the Minister of Education and Science of December 1, 2021. No. 32343. Has a Journal's Unique Identifier: 201159. Scientific disciplines assigned: Physical Culture Sciences (Field of Medical sciences and health sciences); Health Sciences (Field of Medical Sciences and Health Sciences). Punkty Ministerialne z 2019 - aktualny rok 40 punktów. Zalącznik do komunikatu Ministra Edukacji i Nauki z dnia 1 grudnia 2021 r. Lp. 32343. Posiada Unikatowy Identyfikator Czasopisma: 201159. Przypisane dyscypliny naukowe:Nauki o kulturze fizycznej (Dziedzina nauk medycznych i nauk o zdrowiu); Nauki o zdrowiu (Dziedzina nauk medycznych i nauk o zdrowiu). (c) The Authors 2021; Nicolaus Copernicus University in Torun, Polan

Open Access. This article is distributed under the terms of the Creative Commons Attribution Noncommercial License which permits any noncommercial use, distribution, and reproduction in any medium, provided the original author (s) and source are credited. This is an open access article licensed under the terms of the Creative Commons Attribution Non commercial license Share alike. The authors declare that there is no conflict of interests regarding the publication of this paper. Received: 15.11.2021. Revised: 25.11.2021. Accepted: 11.12.2021.

\title{
THE PARAMETERS OF GAS DISCHARGE VISUALIZATION (BIOPHOTONICS) CORRELATED WITH PARAMETERS OF ACUPUNCTURE POINTS, EEG, HRV AND HORMONES
}

\author{
Valeriy Y. Babelyuk ${ }^{1,2}$, Ruslan G. Tserkovniuk ${ }^{1}$, Nazariy V. Babelyuk ${ }^{2}$, Xawery Zukow, ${ }^{3}$, \\ Sofiya V. Ruzhylo ${ }^{4}$, Galyna I. Dubkova ${ }^{2}$, Tetyana A. Korolyshyn ${ }^{1,2,5}$, \\ Viktor Y. Hubyts'kyi ${ }^{1,2}$, Volodymyr V. Kikhtan ${ }^{2}$, Anatoliy I. Gozhenko ${ }^{1}$, \\ Igor L. Popovych ${ }^{1,5}$ \\ ${ }^{1}$ Ukrainian Scientific Research Institute of Medicine for Transport MHU, Odesa, \\ Ukraine valeriybabelyk64@gmail.com prof.gozhenko@gmail.com \\ ${ }^{2}$ Clinical Sanatorium „Moldova”, Truskavets', Ukraine dubkovahalyna@gmail.com \\ ${ }^{3}$ Medical University of Bialystok, Bialystok, Poland xaweryzukow@gmail.com \\ ${ }^{4}$ Ivan Franko Pedagogical University, Drohobych, Ukraine doctor-0701@ukr.net \\ ${ }^{5}$ OO Bohomolets' Institute of Physiology NAS, Kyïv, Ukraine i.popovych@biph.kiev.ua
}

\begin{abstract}
Background. Previously we have been shown that exist strong canonical correlation between parameters of GDV and principal neuroendocrine factors of adaptation as well as parameters of leukocytogram, immunity and phagocytosis. This study, conducted on a much expanded contingent, will analyze the relationships between GDV parameters, on the one hand, and the parameters of acupuncture points (APs), EEG, HRV and adaptation hormones, on the other. Material and Methods. We observed twice 31 women and 29 men aged 26-76 years with dysfunction of neuroendocrine-immune complex. In the morning in basal conditions at first registered kirlianogram by the method of GDV by the device "GDV Chamber" ("Biotechprogress", $\mathrm{SPb}, \mathrm{RF}$ ). Than we registered simultaneously EEG and HRV and recorded electrical conductivity of three pairs of Aps. Finally, a blood sample was taken to determine the plasma levels of the main hormones of adaptation: cortisol, testosterone and triiodothyronine. Results processed by method of canonical analysis, using the software package "Statistica 64". Results. The coefficient of canonical correlation between the electrical conductivity of APs and gas-discharge image (GDI) parameters is 0,635 ; between APs and virtual Chakras parameters - 0,614; instead, between APs and GDV parameters as a whole $-0,707$. The autonomic-endocrine constellation is somewhat more strongly associated with GDI parameters than with virtual Chakras parameters $(0,769$ vs 0,712$)$. Additional inclusion of EEG parameters in the neuroendocrine set increases the strength of the canonical
\end{abstract}


correlation to 0,869. Conclusion. The above data, taken together with the previous ones, state that between parameters of neuroendocrine-immune complex and GDV exist strong canonical correlation suggesting suitability of the latter method.

Keywords: Gas Discharge Visualization, Acupuncture Points, EEG, HRV, Cortisol, Testosterone, Triiodothyronine, Relationships.

\section{INTRODUCTION}

The GDV (kirlianogram, biophotonics) method is based on the registration of stimulated emission of photons and electrons from the skin surface. Korotkov KG [11-13] believes that GDV method measures the distribution of electron densities in human systems and organs. These electron densities are the main basis of physiological energy, so there is reason to say that the GDV method allows us to measure the body's potential energy reserve. GDI, taken off without filter, characterizes the functional changes of organism, while taken with a filter characterizes organic changes. At the same time, the GDV method is a bridge between the logical science of the West and the intuitive science of the East. It allows us to represent the same phenomena in different languages, in different systems, to look at the same things from different points of view.

According to Ayurvedic medicine, Chakras are power centers, related to the endocrine glands and neural plexus as well as to some organs. In Puchko LG [16] we read that the first Chakra is related to the testicles and sacral plexus, second Chakra to the ovaries, adrenals and kidneys, third Chakra to spleen, liver and solar plexus, fourth Chakra to thymus, heart and cardiac plexus, fifth Chakra to thyroid and parathyroid glands, sixth Chakra to pituitary gland and brain, seventh Chakra to pineal gland. Chase CR [8] provides a table according to which the first Chakra is associated with adrenals, pelvic nerve plexus, spine, kidneys, bladder, large intestine; second Chakra with testes/ovaries, inferior mesenteric ganglion, ileum, organs of reproduction; third Chakra with [endocrine] pancreas, celiac plexus ganglion, liver, gall bladder, stomach, duodenum, pancreas, spleen; fourth Chakra with thymus, celiac plexus, heart, circulation, vagus nerve; fifth Chakra with thyroid and parathyroid glands, inferior cervical ganglion, lungs, bronchus, larynx, pharynx, large intestine, vagus nerve; sixth Chakra with pituitary and pineal glands, thalamus, hypothalamus, superior cervical ganglion, left brain, lower brain, ears/nose, left eye; seventh Chakra with pineal gland, right brain, upper brain, right eye.

Korotkov KG [12] put forward the concept that each Chakra is associated with a part of the finger. This approach is embodied in the "GDV Chakras" program, which allows us to quantify the state of virtual Chakras.

We have been shown that exist strong canonical correlation between parameters of GDV and principal neuroendocrine factors of adaptation [1] as well as parameters of leukocytogram [5], immunity [3] and phagocytosis [4].

This study, conducted on a much expanded contingent, will analyze the relationships between GDV parameters, on the one hand, and the parameters of acupuncture points, EEG, HRV and adaptation hormones, on the other.

\section{MATERIAL AND METHODS}

The object of observation were 60 volunteers: 31 women and 29 men aged 26-76 years with dysfunction of neuro-endocrine-immune complex and dysmetabolism.

In the morning we registered the kirlianogram (Dubkova GI) by the method of GDV by the device of "GDV Chamber" ("Biotechprogress", SPb, RF). The first base parameter of GDV is Area of gas discharge image (GDI) in Right, Frontal and Left projections registered 
both with and without polyethylene filter. The second base parameter is a coefficient of Shape. The third base parameter of GDI is Entropy. Program estimates also Energy and Asymmetry of virtual Chakras [11-13].

Electrical conductivity recorded (Hubyts'kyi VY) in follow acupuncture points: $\operatorname{Pg}(\mathrm{ND})$, $\mathrm{TR}(\mathrm{X})$ and MC(AVL) at Right and Left side. Used complex "Medissa". For each pair, the Laterality Index (LI) was calculated according to the formula:

LI, $\%=[200 \cdot($ Right - Left $) /($ Right + Left $)]$.

To assess the parameters of heart rate variability (HRV) recorded (Korolyshyn TA) during 7 min electrocardiogram in II lead (software and hardware complex "CardioLab+HRV", KhAI-MEDICA, Kharkiv). For further analysis the following parameters HRV were selected. Temporal parameters (Time Domain Methods): the standard deviation of all NN intervals (SDNN), the square root of the mean of the sum of the squares of differences between adjacent NN intervals (RMSSD), the percent of interval differences of successive NN intervals greater than $50 \mathrm{msec}\left(\mathrm{pNN}_{50}\right)$; Triangular Index $(\mathrm{TNN})$. Spectral parameters (Frequency Domain Methods): power spectral density (PSD) bands of HRV - high-frequency (HF, range $0,4 \div 0,15 \mathrm{~Hz}$ ), low-frequency ( $\mathrm{LF}$, range $0,15 \div 0,04 \mathrm{~Hz}$ ), very low-frequency (VLF, range $0,04 \div 0,015 \mathrm{~Hz}$ ) and ultralow-frequency (ULF, range $0,015 \div 0,003 \mathrm{~Hz}$ ). Calculated classical indexes: LF/HF; (VLF+LF)/HF; LFnu=100\%・LF/(LF+HF) $[6,7,9,18]$. Baevsky's parameters. Heart rate (HR), Mode (Mo), the Amplitude of Mode (AMo), Variational Sweep (MxDMn), Vegetative Balance Index (VBI=AMo/MxDMn), Stress Index $(\mathrm{BSI}=\mathrm{AMo} / 2 \cdot \mathrm{Mo} \bullet \mathrm{MxDMn})$ as well as Baevsky's Activity of Regulatory Systems Index (BARSI) [6].

Simultaneosly EEG recorded (TA Korolyshyn) a hardware-software complex "NeuroCom Standard" (KhAI MEDICA, Kharkiv) monopolar in 16 loci (Fp1, Fp2, F3, F4, F7, F8, C3, C4, T3, T4, P3, P4, T5, T6, O1, O2) by 10-20 international system, with the reference electrodes $\mathrm{A}$ and Ref tassels on the ears. The duration of the epoch was $25 \mathrm{sec}$. Among the options considered the average EEG amplitude $(\mu \mathrm{V})$, average frequency $(\mathrm{Hz})$, frequency deviation $(\mathrm{Hz})$, index $(\%)$ as well as absolute $\left(\mu \mathrm{V}^{2} / \mathrm{Hz}\right)$ and relative $(\%)$ PSD of basic rhythms: $\beta(35 \div 13 \mathrm{~Hz}), \alpha(13 \div 8 \mathrm{~Hz}), \theta(8 \div 4 \mathrm{~Hz})$ and $\delta(4 \div 0,5 \mathrm{~Hz})$ in all loci, according to the instructions of the device. In addition, calculated coefficient of Asymmetry (As) and Laterality Index (LI) for PSD each Rhythm using formulas:

As, $\%=100 \cdot(\operatorname{Max}-\mathrm{Min}) / \mathrm{Min}$;

LI, $\%=\Sigma[200 \bullet($ Right - Left $) /($ Right + Left $)] / 8$

We calculated also for each locus EEG and HRV Shannon's [18] Entropy (h) of normalized PSD using Popovych's formulas [14]:

$\mathrm{hEEG}=-\left[\mathrm{PSD} \alpha \bullet \log _{2} \mathrm{PSD} \alpha+\mathrm{PSD} \beta \bullet \log _{2} \mathrm{PSD} \beta+\mathrm{PSD} \theta \bullet \log _{2} \mathrm{PSD} \theta+\mathrm{PSD} \delta \bullet \log _{2} \mathrm{PSD} \delta\right] / \log _{2} 4$

$\mathrm{hHRV}=-\left[\mathrm{PSHF} \bullet \log _{2} \mathrm{PSHF}+\mathrm{PSLF} \bullet \log _{2} \mathrm{PSLF}+\mathrm{PSVLF} \bullet \log _{2} \mathrm{PSVLF}+\mathrm{PSULF} \bullet \log _{2} \mathrm{PSULF}\right] / \log _{2} 4$

Among hormones determined (Kikhtan VV) major adaptation hormones Cortisol, Testosterone and Triiodothyronine (by the ELISA with the use of analyzer "RT-2100C" and corresponding sets of reagents from “Алкор Био”, XЕMA Co., Ltd and DRG International Inc.).

\section{RESULTS AND DISCUSSION}

At the first stage of the analysis, a matrix of correlations between the parameters of acupuncture points (AP) and gas-discharge image (GDI) was created (Table 1).

The next step was to calculate the canonical correlation between the two sets of parameters. The factor structure of acupuncture root is formed by indicators of electrical conductivity, but not its lateralization. GDI-root represents the coefficients of the shape of the image recorded without a filter in all three projections, and the entropy of the image also in all 
projections, but taken using a polyethylene filter, as well as the Korotkov's activation coefficient. These parameters carry factor loads with the same sign as the conductivity parameters. Instead, the symmetry of GDI and its area in all projections are related to electrical conductivity in the opposite way. The canonical correlation between the two constellations is significant (Table 2 and Fig. 1).

Following the accepted algorithm, the canonical analysis of the relationship between the parameters of AP and virtual Chakras was performed (Tables 3 and 4). This time, the acupuncture root factor structure included AP MC(AVL) lateralization, which negatively correlates with the Asymmetry of the fifth and sixth virtual Chakras registered with the filter. Interestingly, the Energy of the fifth chakra was outside the structure of the root. In general, the strength of the canonical correlation between AP and Chakras was at the previous level (Fig. 2).

If the GDI and Chakras parameters are combined in one set, its canonical correlation with APs is stronger than in a separate analysis (Table 5 and Fig. 3).

Table 1. Matrix of correlations between the parameters of acupuncture points and gasdischarge image

\begin{tabular}{|c|c|c|c|c|c|c|c|c|c|}
\hline \multirow[b]{3}{*}{ Variable } & \multicolumn{9}{|c|}{$\begin{array}{l}\text { Correlations } \\
\mathrm{N}=119\end{array}$} \\
\hline & $\mathrm{Pg}(\mathrm{ND})$ & $\mathrm{Pg}(\mathrm{ND})$ & $\mathrm{Pg}(\mathrm{ND})$ & MC(AVL) & MC(AVL) & MC(AVL) & $\operatorname{TR}(X)$ & $\operatorname{TR}(\mathrm{X})$ & $\operatorname{TR}(X)$ \\
\hline & R & L & $\mathrm{LI}$ & R & $\mathrm{L}$ & LI & $\mathrm{R}$ & $\mathrm{L}$ & LI \\
\hline KAC & 0,28 & 0,26 & 0,09 & 0,17 & 0,20 & $-0,04$ & 0,24 & 0,32 & 0,00 \\
\hline Area $\mathrm{R}$ & $-0,09$ & $-0,09$ & 0,00 & $-0,02$ & $-0,09$ & 0,02 & $-0,17$ & $-0,21$ & 0,02 \\
\hline Area RF & 0,07 & 0,02 & 0,07 & 0,15 & 0,07 & $-0,03$ & $-0,00$ & $-0,00$ & 0,01 \\
\hline Entropy R & 0,02 & $-0,03$ & 0,09 & $-0,01$ & $-0,07$ & $-0,00$ & 0,09 & 0,05 & $-0,05$ \\
\hline Entropy RF & 0,11 & 0,10 & 0,02 & 0,16 & 0,20 & $-0,10$ & 0,08 & 0,14 & $-0,13$ \\
\hline CR & 0,15 & 0,16 & 0,03 & 0,05 & 0,15 & $-0,05$ & 0,19 & 0,29 & $-0,02$ \\
\hline CRF & 0,02 & 0,05 & $-0,01$ & $-0,10$ & $-0,02$ & $-0,05$ & 0,08 & 0,07 & $-0,04$ \\
\hline Area $\mathrm{F}$ & $-0,09$ & $-0,12$ & 0,06 & $-0,04$ & $-0,12$ & 0,01 & $-0,19$ & $-0,24$ & 0,02 \\
\hline Area FF & 0,08 & 0,03 & 0,08 & 0,14 & 0,07 & $-0,02$ & 0,05 & 0,04 & 0,02 \\
\hline Symmetry & $-0,23$ & $-0,25$ & $-0,08$ & $-0,16$ & $-0,27$ & 0,04 & $-0,26$ & $-0,39$ & $-0,01$ \\
\hline Symmetry F & $-0,11$ & $-0,10$ & $-0,02$ & $-0,00$ & $-0,07$ & 0,05 & $-0,07$ & $-0,10$ & 0,07 \\
\hline Entropy F & 0,07 & 0,03 & 0,09 & $-0,04$ & $-0,09$ & $-0,03$ & 0,05 & 0,02 & $-0,05$ \\
\hline Entropy FF & 0,18 & 0,19 & $-0,10$ & 0,24 & 0,28 & $-0,05$ & 0,16 & 0,21 & $-0,18$ \\
\hline $\mathrm{CF}$ & 0,19 & 0,22 & $-0,01$ & 0,12 & 0,24 & $-0,06$ & 0,24 & 0,36 & 0,01 \\
\hline CFF & 0,06 & 0,08 & $-0,05$ & $-0,02$ & 0,04 & $-0,02$ & 0,13 & 0,12 & 0,01 \\
\hline Area L & $-0,12$ & $-0,15$ & 0,02 & $-0,09$ & $-0,18$ & 0,01 & $-0,23$ & $-0,29$ & 0,00 \\
\hline Area LF & 0,06 & 0,01 & 0,08 & 0,07 & $-0,03$ & 0,04 & $-0,06$ & $-0,08$ & 0,01 \\
\hline Entropy L & 0,06 & 0,02 & 0,04 & 0,09 & 0,03 & 0,02 & 0,04 & $-0,02$ & 0,05 \\
\hline Entropy LF & 0,17 & 0,20 & $-0,12$ & 0,19 & 0,25 & $-0,16$ & 0,17 & 0,22 & $-0,18$ \\
\hline $\mathrm{CL}$ & 0,19 & 0,22 & $-0,00$ & 0,14 & 0,24 & $-0,00$ & 0,24 & 0,35 & 0,02 \\
\hline CLF & 0,00 & 0,04 & $-0,09$ & $-0,05$ & 0,05 & $-0,11$ & 0,12 & 0,14 & 0,00 \\
\hline
\end{tabular}

Note. For a sample with $\mathrm{n}=119_{0,05}|\mathbf{r}|>0,18 ;{ }_{0,01}|\mathbf{r}|>0,24 ;_{0,001}|\mathbf{r}|>0,31$. 
Table 2. Factor structure of electrical conductivity and gas-discharge image

\begin{tabular}{|l|c|}
\hline Left set & $\mathbf{R}$ \\
\hline $\mathrm{TR}(\mathrm{X}) \mathrm{L}$ & $\mathbf{- 0 , 7 3 9}$ \\
\hline $\mathrm{MC}(\mathrm{AVL}) \mathrm{L}$ & $\mathbf{- 0 , 6 3 3}$ \\
\hline $\mathrm{TR}(\mathrm{X}) \mathrm{R}$ & $\mathbf{- 0 , 5 0 0}$ \\
\hline $\mathrm{Pg}(\mathrm{ND}) \mathrm{L}$ & $\mathbf{- 0 , 4 5 5}$ \\
\hline $\mathrm{MC}(\mathrm{AVL}) \mathrm{R}$ & $\mathbf{- 0 , 3 8 5}$ \\
\hline Pg(ND) R & $\mathbf{- 0 , 3 8 4}$ \\
\hline Right set & $\mathbf{R}$ \\
\hline Shape Coeff F & $\mathbf{- 0 , 7 7 3}$ \\
\hline Shape Coeff L & $\mathbf{- 0 , 7 3 1}$ \\
\hline Shape Coeff R & $\mathbf{- 0 , 6 1 7}$ \\
\hline Korotkov's AC & $\mathbf{- 0 , 5 3 8}$ \\
\hline Entropy L (f) & $\mathbf{- 0 , 4 0 9}$ \\
\hline Entropy F (f) & $\mathbf{- 0 , 3 9 5}$ \\
\hline Entropy R (f) & $\mathbf{- 0 , 3 8 0}$ \\
\hline Symmetry & $\mathbf{0 , 8 5 0}$ \\
\hline Area Left & $\mathbf{0 , 5 6 7}$ \\
\hline Area Frontal & $\mathbf{0 , 4 7 1}$ \\
\hline Area Right & $\mathbf{0 , 4 0 7}$ \\
\hline
\end{tabular}

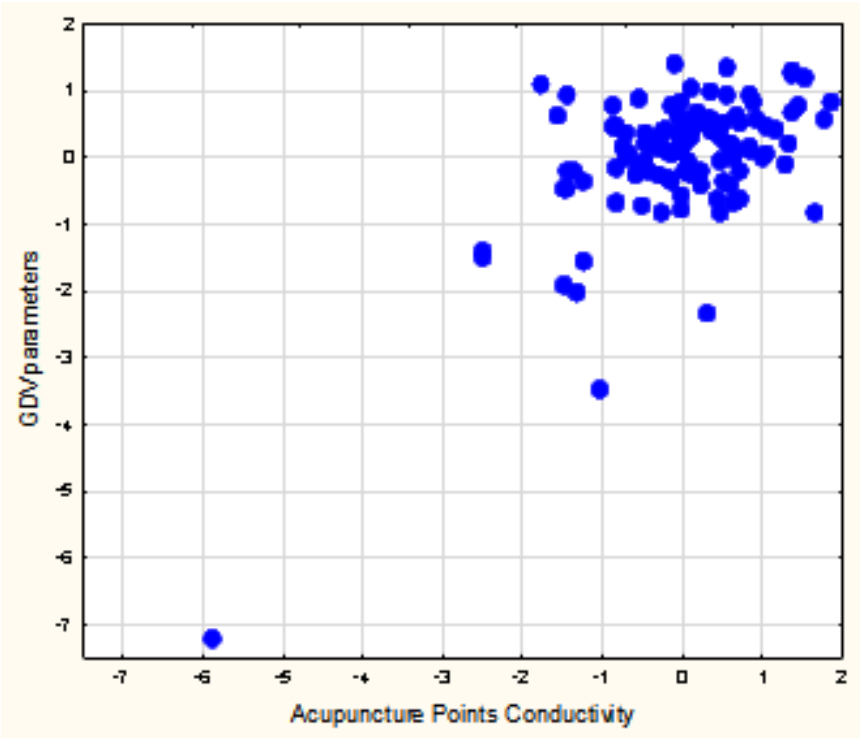

$\mathrm{R}=0,635 ; \mathrm{R}^{2}=0,404 ; \chi_{(88)}^{2}=135 ; \mathrm{p}=0,001 ; \Lambda$ Prime $=0,288$

Fig. 1. Scatterplot of canonical correlation between electrical conductivity of Acupuncture Points (X-line) and GDI parameters (Y-line) 
Table 3. Matrix of correlations between the parameters of acupuncture points and virtual Chakras

\begin{tabular}{|l|r|r|r|r|r|r|r|r|r|}
\hline \multirow{2}{*}{$\begin{array}{l}\text { Root } \\
\text { Variable }\end{array}$} & \multicolumn{7}{|l|}{ Correlations, left set with right set } \\
\cline { 2 - 11 } & CH1E & CH2E & CH3E & CH4E & $\begin{array}{c}\text { CH6E } \\
\text { F }\end{array}$ & CH7E & $\begin{array}{c}\text { CH5A } \\
\text { F }\end{array}$ & $\begin{array}{c}\text { CH6 } \\
\text { A }\end{array}$ & $\begin{array}{c}\text { CH6A } \\
\text { F }\end{array}$ \\
\hline Pg(ND)R & $-0,06$ & $-0,134$ & $-0,08$ & $-0,09$ & 0,20 & $-0,05$ & $-0,04$ & 0,10 & 0,00 \\
\hline Pg(ND)L & $-0,06$ & $-0,147$ & $-0,11$ & $-0,14$ & 0,17 & $-0,05$ & 0,03 & 0,13 & 0,07 \\
\hline MC(AVL)R & $-0,02$ & $-0,138$ & $-0,07$ & $-0,13$ & 0,10 & $-0,06$ & 0,03 & 0,14 & 0,05 \\
\hline MC(AVL)L & $-0,09$ & $-0,227$ & $-0,16$ & $-0,23$ & 0,05 & $-0,12$ & 0,16 & 0,20 & 0,16 \\
\hline TR(X) & $-0,13$ & $-0,264$ & $-0,22$ & $-0,19$ & 0,07 & $-0,21$ & 0,17 & 0,18 & 0,20 \\
\hline TR(X) & $-0,21$ & $-0,342$ & $-0,28$ & $-0,28$ & 0,05 & $-0,23$ & 0,19 & 0,18 & 0,22 \\
\hline MC(AVL)LI & 0,01 & 0,070 & 0,02 & 0,03 & $-0,02$ & 0,05 & $-0,26$ & $-0,01$ & $-0,24$ \\
\hline
\end{tabular}

Table 4. Factor structure of electrical conductivity and virtual Chakras

\begin{tabular}{|l|c|}
\hline Left set & $\mathbf{R}$ \\
\hline $\mathrm{TR}(\mathrm{X}) \mathrm{L}$ & $\mathbf{- 0 , 7 1 8}$ \\
\hline $\mathrm{MC}(\mathrm{AVL}) \mathrm{L}$ & $\mathbf{- 0 , 6 0 5}$ \\
\hline $\mathrm{TR}(\mathrm{X}) \mathrm{R}$ & $\mathbf{- 0 , 4 8 1}$ \\
\hline $\mathrm{Pg}(\mathrm{ND}) \mathrm{L}$ & $\mathbf{- 0 , 4 1 0}$ \\
\hline $\mathrm{MC}(\mathrm{AVL}) \mathrm{R}$ & $\mathbf{- 0 , 3 4 6}$ \\
\hline $\mathrm{Pg}(\mathrm{ND}) \mathrm{R}$ & $\mathbf{- 0 , 2 9 3}$ \\
\hline $\mathrm{MC}(\mathrm{AVL}) \mathrm{LI}$ & $\mathbf{0 , 3 3 0}$ \\
\hline Right set & $\mathbf{R}$ \\
\hline Ch2 E & $\mathbf{0 , 6 5 3}$ \\
\hline Ch4 E & $\mathbf{0 , 6 3 3}$ \\
\hline Ch3 E & $\mathbf{0 , 5 5 0}$ \\
\hline Ch1 E & $\mathbf{0 , 4 6 6}$ \\
\hline Ch7 E & $\mathbf{0 , 4 1 5}$ \\
\hline Ch6 E (f) & $\mathbf{0 , 1 2 4}$ \\
\hline Ch6 A & $\mathbf{- 0 , 5 3 0}$ \\
\hline Ch5 A (f) & $\mathbf{- 0 , 5 7 0}$ \\
\hline Ch6 A (f) & $\mathbf{- 0 , 5 3 0}$ \\
\hline
\end{tabular}

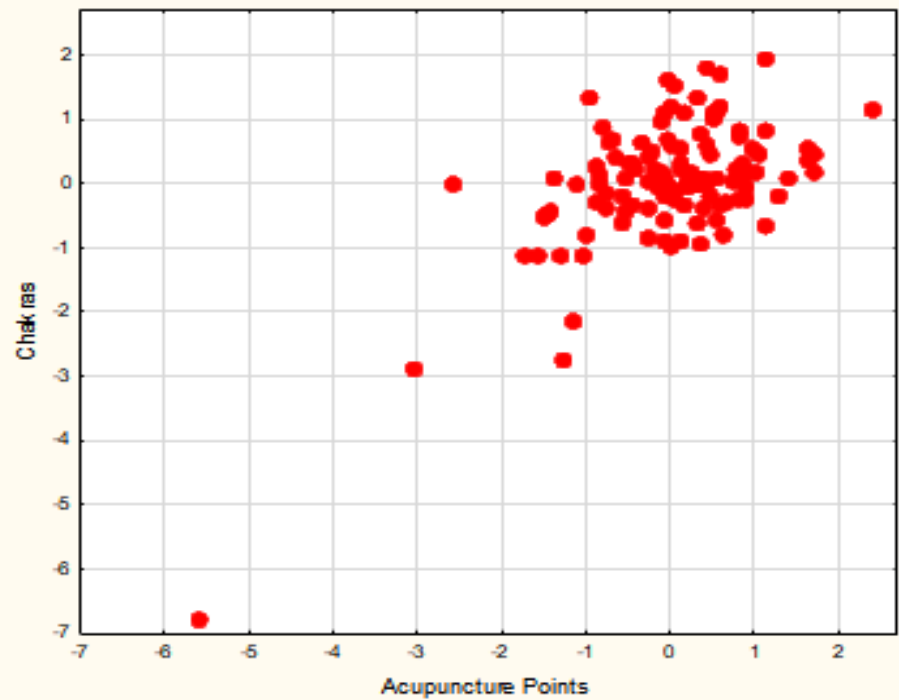

$\mathrm{R}=0,614 ; \mathrm{R}^{2}=0,377 ; \chi^{2}{ }_{(63)}=114 ; \mathrm{p}<10^{-4} ; \Lambda$ Prime $=0,353$

Fig. 2. Scatterplot of canonical correlation between electrical conductivity of Acupuncture Points (X-line) and virtual Chakras parameters (Y-line) 
Table 5. Factor structure of electrical conductivity and virtual Chakras Roots

\begin{tabular}{|l|c|}
\hline Left set & $\mathbf{R}$ \\
\hline TR(X) L & $\mathbf{- 0 , 7 0 6}$ \\
\hline MC(AVL) L & $\mathbf{- 0 , 6 2 0}$ \\
\hline TR(X) R & $\mathbf{- 0 , 4 8 5}$ \\
\hline Pg(ND) L & $\mathbf{- 0 , 3 6 6}$ \\
\hline MC(AVL) R & $\mathbf{- 0 , 3 6 5}$ \\
\hline Pg(ND) R & $\mathbf{- 0 , 2 8 9}$ \\
\hline MC(AVL) LI & $\mathbf{0 , 3 4 9}$ \\
\hline Right set & $\mathbf{R}$ \\
\hline Symmetry & $\mathbf{0 , 6 8 2}$ \\
\hline Ch2 E & $\mathbf{0 , 5 7 9}$ \\
\hline Ch4 E & $\mathbf{0 , 5 4 6}$ \\
\hline Ch3 E & $\mathbf{0 , 4 8 3}$ \\
\hline Area Left & $\mathbf{0 , 4 6 9}$ \\
\hline Ch1 E & $\mathbf{0 , 4 1 6}$ \\
\hline Korotkov's AC & $\mathbf{0 , 4 1 4}$ \\
\hline Area Frontal & $\mathbf{0 , 3 8 7}$ \\
\hline Ch7 E & $\mathbf{0 , 3 7 8}$ \\
\hline Area Right & $\mathbf{0 , 3 3 4}$ \\
\hline Ch6 E (f) & $\mathbf{0 , 1 1 7}$ \\
\hline Shape Coeff F & $\mathbf{- 0 , 6 2 9}$ \\
\hline Shape Coeff L & $\mathbf{- 0 , 5 8 6}$ \\
\hline Ch5 A (f) & $\mathbf{- 0 , 4 9 9}$ \\
\hline Shape Coeff R & $\mathbf{- 0 , 4 9 6}$ \\
\hline Ch6 A (f) & $\mathbf{- 0 , 4 5 7}$ \\
\hline Entropy L (f) & $\mathbf{- 0 , 3 6 1}$ \\
\hline Entropy F (f) & $\mathbf{- 0 , 3 3 4}$ \\
\hline Entropy R (f) & $\mathbf{- 0 , 3 2 6}$ \\
\hline Ch6 A & $\mathbf{- 0 , 2 8 1}$ \\
\hline
\end{tabular}

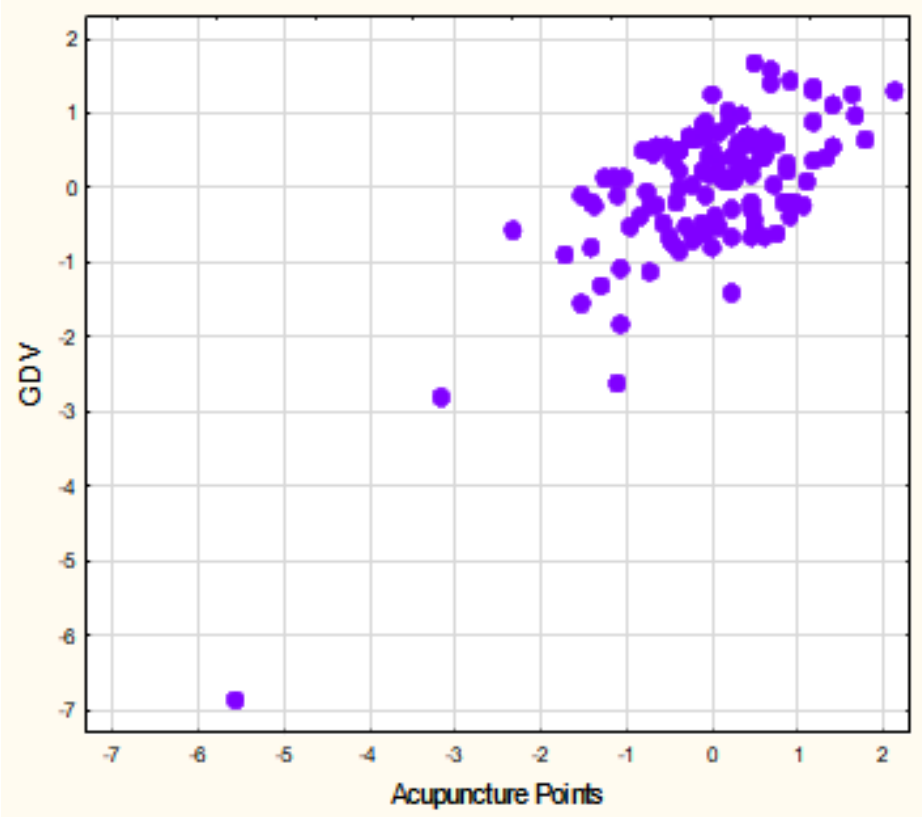

$\mathrm{R}=0,707 ; \mathrm{R}^{2}=0,499 ; \chi_{(140)}^{2}=178 ; \mathrm{p}=0,016 ; \Lambda$ Prime $=0,180$

Fig. 3. Scatterplot of canonical correlation between electrical conductivity of Acupuncture Points (X-line) and total GDV parameters (Y-line) 
Screening of correlations between GDI and HRV parameters revealed (Table 6) that the old good rhythm frequency (Heart Rate) has the maximum coefficients for sampling, correlating positively with GDI entropy in all projections under both recording conditions. Negative correlation with entropy was found for markers of vagal tone (RMSSD, $\mathrm{pNN}_{50}, \mathrm{HF}$ ), with the maximum with the entropy of GDI recorded with a filter in frontal projection. Markers of sympathetic tone (LFnu, LF/HF, LF\%) correlate, naturally, positively. In addition, the latter correlate positively with the shape coefficients and negatively with the GDI area in the left projection.

Table 6. Matrix of correlations between the autonomic-endocrine and GDI parameters

\begin{tabular}{|c|c|c|c|c|c|c|c|c|c|c|c|c|c|}
\hline \multirow[b]{2}{*}{ Variabli } & \multicolumn{13}{|c|}{$\begin{array}{l}\text { Correlations } \\
\mathrm{N}=119\end{array}$} \\
\hline & $\begin{array}{c}\text { Test } \\
\text { act }\end{array}$ & $\begin{array}{l}\text { Test } \\
\text { stand }\end{array}$ & $\begin{array}{c}\text { BSI } \\
\text { In }\end{array}$ & HR & $\begin{array}{l}\text { RMS } \\
\text { SD }\end{array}$ & $\begin{array}{c}\mathrm{pNN} \\
50\end{array}$ & LF & $\mathrm{HF}$ & $\begin{array}{l}\mathrm{LF} / \\
\mathrm{HF}\end{array}$ & $\mathrm{Cl}$ & $\begin{array}{l}\text { LF } \\
\%\end{array}$ & LFnu & $\begin{array}{c}\text { VLF } \\
\%\end{array}$ \\
\hline KAC & 0,04 & $-0,05$ & $-0,05$ & 0,14 & $-0,02$ & $-0,01$ & 0,15 & $-0,04$ & 0,24 & 0,09 & 0,25 & 0,26 & $-0,16$ \\
\hline ER & $-0,09$ & 0,18 & 0,17 & 0,33 & $-0,13$ & $-0,13$ & $-0,10$ & $-0,09$ & 0,17 & 0,06 & 0,20 & 0,06 & $-0,18$ \\
\hline ER f & $-0,03$ & 0,02 & 0,09 & 0,28 & $-0,17$ & $-0,22$ & $-0,12$ & $-0,15$ & 0,09 & 0,04 & 0,20 & 0,05 & $-0,19$ \\
\hline SCR & 0,05 & $-0,02$ & $-0,09$ & 0,06 & $-0,00$ & $-0,01$ & 0,16 & $-0,04$ & 0,19 & 0,06 & 0,18 & 0,22 & $-0,08$ \\
\hline SCR f & 0,01 & 0,05 & 0,01 & 0,04 & $-0,08$ & $-0,10$ & 0,02 & $-0,14$ & 0,14 & 0,02 & 0,12 & 0,20 & $-0,04$ \\
\hline $\mathrm{AF}$ & 0,13 & 0,06 & 0,14 & 0,01 & $-0,09$ & $-0,06$ & $-0,20$ & $-0,04$ & $-0,10$ & 0,02 & 0,15 & $-0,12$ & 0,11 \\
\hline AF $f$ & 0,27 & $-0,03$ & 0,11 & 0,08 & $-0,11$ & $-0,07$ & $-0,17$ & $-0,07$ & 0,03 & 0,07 & 0,02 & $-0,02$ & 0,02 \\
\hline$E F$ & $-0,06$ & 0,06 & 0,09 & 0,22 & $-0,12$ & $-0,14$ & $-0,07$ & $-0,12$ & 0,10 & 0,06 & 0,11 & 0,08 & $-0,08$ \\
\hline$E F$ f & $-0,08$ & $-0,12$ & 0,19 & 0,32 & $-0,22$ & $-0,23$ & $-0,15$ & $-0,24$ & 0,21 & 0,20 & 0,20 & 0,22 & $-0,13$ \\
\hline SCF & 0,07 & $-0,07$ & $-0,09$ & 0,09 & $-0,03$ & $-0,04$ & 0,14 & $-0,07$ & 0,20 & 0,07 & 0,19 & 0,23 & $-0,09$ \\
\hline SCF $\mathrm{f}$ & 0,07 & $-0,07$ & 0,01 & 0,07 & $-0,08$ & $-0,10$ & 0,03 & $-0,12$ & 0,18 & 0,09 & 0,10 & 0,21 & $-0,02$ \\
\hline $\mathrm{AL}$ & 0,02 & 0,16 & 0,13 & 0,01 & $-0,03$ & $-0,03$ & $-0,20$ & $-0,01$ & $-0,22$ & $-0,05$ & 0,22 & $-0,23$ & 0,13 \\
\hline EL & 0,05 & 0,21 & 0,03 & 0,26 & $-0,18$ & $-0,21$ & $-0,16$ & $-0,20$ & 0,20 & 0,14 & 0,09 & 0,10 & $-0,05$ \\
\hline ELF & $-0,06$ & 0,11 & 0,02 & 0,22 & $-0,15$ & $-0,20$ & $-0,11$ & $-0,19$ & 0,05 & 0,07 & 0,08 & 0,08 & $-0,05$ \\
\hline $\mathrm{SCL}$ & 0,10 & $-0,09$ & $-0,09$ & 0,11 & $-0,04$ & $-0,05$ & 0,15 & $-0,07$ & 0,24 & 0,08 & 0,25 & 0,27 & $-0,14$ \\
\hline
\end{tabular}

Table 7. Factor structure of HRV\&endocrine and GDI Roots

\begin{tabular}{|c|c|}
\hline Left set & $\mathbf{R}$ \\
\hline Testosterone, nM/L & $-0,616$ \\
\hline LFnu, \% & $-0,283$ \\
\hline $\mathrm{LF} / \mathrm{HF}$ & $-0,214$ \\
\hline$(\mathrm{VLF}+\mathrm{LF}) / \mathrm{HF}$ & $-0,208$ \\
\hline$(\mathrm{VLF}+\mathrm{ULF}) / \mathrm{TP}$ & $-0,145$ \\
\hline Heart Rate & $-0,142$ \\
\hline Stress Index, ln un & $-0,093$ \\
\hline Testosterone, $\mathrm{Z}$ & 0,525 \\
\hline RMSSD, msec & 0,123 \\
\hline $\mathrm{HF}, \mathrm{msec}^{2}$ & 0,097 \\
\hline $\mathrm{LF}, \mathrm{msec}^{2}$ & 0,052 \\
\hline $\mathrm{pNN}_{50}, \%$ & 0,009 \\
\hline Right set & $\mathbf{R}$ \\
\hline Entropy R & 0,370 \\
\hline Entropy L & 0,266 \\
\hline Entropy L (f) & 0,250 \\
\hline Entropy F & 0,201 \\
\hline Entropy R (f) & 0,159 \\
\hline Area Left & 0,109 \\
\hline Shape Coeff R (f) & 0,022 \\
\hline Entropy F (f) & 0,019 \\
\hline
\end{tabular}




\begin{tabular}{|l|c|}
\hline Area Frontal (f) & $\mathbf{- 0 , 3 3 1}$ \\
\hline Shape Coeff L & $\mathbf{- 0 , 1 2 2}$ \\
\hline Area Frontal & $\mathbf{- 0 , 1 2 0}$ \\
\hline Shape Coeff F (f) & $\mathbf{- 0 , 1 1 5}$ \\
\hline Shape Coeff F & $\mathbf{- 0 , 0 7 9}$ \\
\hline Korotkov's AC & $\mathbf{- 0 , 0 3 7}$ \\
\hline Shape Coeff R & $\mathbf{- 0 , 0 2 1}$ \\
\hline
\end{tabular}

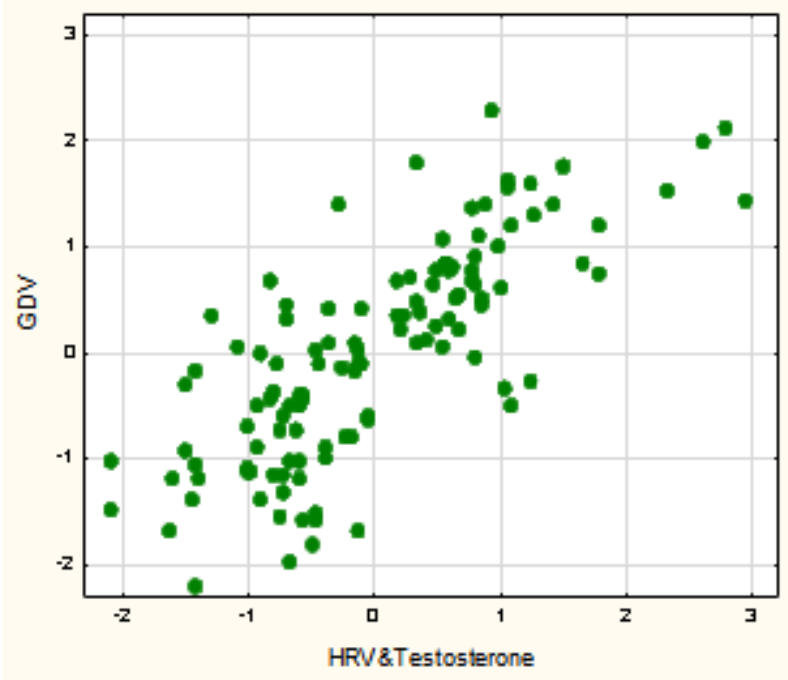

\section{$\mathrm{R}=0,769 ; \mathrm{R}^{2}=0,591 ; \chi^{2}{ }_{(210)}=282 ; \mathrm{p}=0,0007 ; \Lambda$ Prime $=0,065$}

Fig. 4. Scatterplot of canonical correlation between HRV\&Endocrine (X-line) and GDV (Y-line) parameters

Among the detected hormones, only testosterone significantly correlates with GDI parameters. Interestingly, actual testosterone levels correlate positively with the area of GDI recorded with the filter, while standardized by sex and age with the entropy of GDI recorded without the filter in the left projection.

The activation coefficient proposed by Korotkov KG as a GDV Stress Index, according to our data, does not correlate with the Baevsky's RM HRV Stress Index, and the correlation with markers of sympathetic-vagal balance and sympathetic tone, although statistically significant, is not convincing enough.

In general, the canonical correlation between the analyzed constellations is strong (Table 7 and Fig. 4).

Instead, as a result of the canonical correlation with the parameters of the virtual Chakras, cortisol and triiodothyronine were included in the factor structure of the autonomicendocrine root (Tables 8 and 9). The prominent place in it of the VLF band encourages us to dwell in more detail on the interpretation of its physiological essence.

As noted Shaffer F \& Ginsberg JP [18], there is uncertainty regarding the physiological mechanisms responsible for activity within the VLF $(0,04 \div 0,0033 \mathrm{~Hz})$ band. The heart's intrinsic nervous system appears to contribute to the VLF rhythm and the sympathetic nervous system influences the amplitude and frequency of its oscillations. VLF power may also be generated by physical activity, thermoregulatory, renin-angiotensin, and endothelial influences on the heart. Vagal activity may contribute to VLF power since parasympathetic blockade almost completely abolishes it. In contrast, sympathetic blockade does not affect VLF power. The VLF rhythm appears to be generated by the stimulation of afferent sensory neurons in the heart. This, in turn, activates various levels of the feedback and feed-forward 
loops in the heart's intrinsic cardiac nervous system, as well as between the heart, the extrinsic cardiac ganglia, and spinal column. This experimental evidence suggests that the heart intrinsically generates the VLF rhythm and efferent sympathetic nervous system activity due to physical activity and stress responses modulates its amplitude and frequency. Because in our device ULF band (range $0,015 \div 0,003 \mathrm{~Hz}$ ) is integrated into the lower zone of VLF band, what has been said about the latter also applies to the former. By the way, the relative PS of these bands during the analysis were combined into an option (VLF+ULF).

Table 8. Matrix of correlations between the autonomic-endocrine parameters and parameters of virtual Chakras

\begin{tabular}{|c|c|c|c|c|c|c|c|c|c|c|c|c|c|c|}
\hline $\begin{array}{l}\text { Chakra } \\
\text { E\&A }\end{array}$ & $\begin{array}{l}\text { Test } \\
\text { raw }\end{array}$ & $\begin{array}{l}\text { Test } \\
\text { stand }\end{array}$ & $\begin{array}{l}\text { Cor- } \\
\text { tisol }\end{array}$ & T3 & $\begin{array}{l}\text { AM } \\
\text { o }\end{array}$ & $\mathrm{dX}$ & $\begin{array}{l}\mathrm{AMo} / \\
\mathrm{dX}\end{array}$ & BSI & HR & $\begin{array}{l}\mathrm{HF} \\
\%\end{array}$ & $\begin{array}{l}\mathrm{LF} \\
\mathrm{nu}\end{array}$ & $\begin{array}{l}\text { VLF } \\
\text { ULF }\end{array}$ & VLF & TP \\
\hline $1 \mathrm{E} \mathrm{f}$ & $-0,02$ & 0,15 & $-0,01$ & $-0,08$ & $-0,04$ & $-0,02$ & $-0,03$ & 0,01 & 0,23 & 0,10 & $-0,09$ & 0,12 & 0,14 & 0,09 \\
\hline $2 \mathrm{E} \mathrm{f}$ & $-0,21$ & 0,08 & $-0,06$ & $-0,02$ & $-0,06$ & 0,02 & $-0,06$ & $-0,03$ & 0,15 & 0,17 & $-0,13$ & 0,07 & 0,08 & 0,05 \\
\hline $3 \mathrm{E}$ & $-0,13$ & 0,10 & $-0,04$ & 0,02 & 0,07 & $-0,09$ & 0,06 & 0,06 & 0,06 & 0,17 & $-0,21$ & 0,11 & 0,11 & 0,07 \\
\hline $4 \mathrm{E}$ & $-0,23$ & 0,13 & $-0,01$ & $-0,00$ & 0,01 & $-0,08$ & 0,03 & 0,04 & 0,09 & 0,14 & $-0,18$ & 0,06 & 0,07 & 0,02 \\
\hline $4 \mathrm{E} \mathrm{f}$ & $-0,18$ & 0,12 & $-0,07$ & $-0,08$ & $-0,07$ & 0,01 & $-0,07$ & $-0,03$ & 0,19 & 0,15 & $-0,08$ & $-0,07$ & $-0,06$ & $-0,07$ \\
\hline $5 \mathrm{E}$ & 0,08 & 0,11 & $-0,01$ & $-0,01$ & 0,19 & $-0,18$ & 0,19 & 0,22 & 0,21 & 0,02 & $-0,04$ & $-0,04$ & $-0,02$ & $-0,08$ \\
\hline $5 \mathrm{E} \mathrm{f}$ & 0,07 & 0,08 & $-0,02$ & $-0,07$ & 0,14 & $-0,13$ & 0,12 & 0,14 & 0,20 & 0,05 & $-0,01$ & $-0,06$ & $-0,05$ & $-0,09$ \\
\hline $6 \mathrm{E}$ & 0,01 & 0,23 & $-0,00$ & 0,01 & 0,22 & $-0,19$ & 0,22 & 0,24 & 0,20 & 0,04 & $-0,07$ & $-0,02$ & $-0,01$ & $-0,07$ \\
\hline $6 \mathrm{Ef}$ & $-0,01$ & 0,14 & $-0,02$ & $-0,05$ & 0,16 & $-0,14$ & 0,15 & 0,18 & 0,22 & 0,08 & $-0,04$ & $-0,03$ & $-0,01$ & $-0,05$ \\
\hline $7 \mathrm{E}$ & $-0,16$ & 0,17 & $-0,01$ & 0,02 & 0,08 & $-0,10$ & 0,07 & 0,07 & 0,16 & 0,16 & $-0,18$ & 0,15 & 0,17 & 0,10 \\
\hline $7 \mathrm{E} \mathrm{f}$ & $-0,19$ & 0,11 & $-0,07$ & $-0,02$ & $-0,02$ & $-0,02$ & $-0,02$ & 0,03 & 0,25 & 0,18 & $-0,10$ & 0,04 & 0,05 & 0,03 \\
\hline $1 \mathrm{~A} \mathrm{f}$ & 0,01 & $-0,23$ & 0,05 & $-0,04$ & 0,23 & $-0,28$ & 0,24 & 0,24 & 0,05 & $-0,10$ & 0,03 & 0,21 & 0,24 & 0,14 \\
\hline $2 \mathrm{~A}$ & 0,01 & 0,07 & $-0,20$ & 0,02 & $-0,12$ & 0,11 & $-0,12$ & $-0,13$ & $-0,06$ & 0,13 & $-0,04$ & $-0,01$ & $-0,01$ & 0,01 \\
\hline $2 \mathrm{Aff}$ & 0,08 & $-0,06$ & 0,04 & $-0,04$ & $-0,10$ & 0,11 & $-0,14$ & $-0,15$ & $-0,10$ & 0,32 & $-0,28$ & 0,07 & 0,07 & 0,11 \\
\hline $3 \mathrm{~A}$ & 0,19 & 0,02 & $-0,17$ & $-0,03$ & $-0,12$ & 0,07 & $-0,18$ & $-0,16$ & $-0,09$ & $-0,07$ & 0,10 & $-0,13$ & $-0,11$ & $-0,12$ \\
\hline $4 \mathrm{~A}$ & 0,15 & 0,10 & 0,07 & $-0,03$ & 0,15 & $-0,22$ & 0,14 & 0,13 & 0,11 & $-0,12$ & 0,05 & 0,16 & 0,16 & 0,07 \\
\hline $4 \mathrm{~A} \mathrm{f}$ & $-0,06$ & 0,00 & 0,03 & 0,00 & $-0,05$ & 0,01 & $-0,05$ & $-0,06$ & 0,09 & $-0,06$ & $-0,02$ & 0,25 & 0,26 & 0,24 \\
\hline $5 \mathrm{~A}$ & $-0,02$ & $-0,24$ & 0,09 & 0,24 & 0,15 & $-0,10$ & $\mathbf{0 , 2 0}$ & 0,18 & $-0,01$ & $-0,07$ & 0,11 & $-0,05$ & $-0,05$ & $-0,06$ \\
\hline $5 \mathrm{Af}$ & 0,04 & $-0,31$ & 0,02 & 0,07 & 0,08 & $-0,09$ & 0,07 & 0,05 & $-0,01$ & $-0,03$ & 0,02 & $-0,02$ & $-0,01$ & $-0,02$ \\
\hline $6 \mathrm{~A}$ & $-0,09$ & $-0,34$ & 0,08 & 0,20 & 0,14 & $-0,13$ & 0,19 & 0,17 & $-0,00$ & $-0,04$ & 0,11 & $-0,02$ & $-0,01$ & $-0,03$ \\
\hline $6 \mathrm{Af}$ & 0,07 & $-0,35$ & $-0,00$ & 0,03 & 0,06 & $-0,07$ & 0,04 & 0,03 & 0,01 & $-0,04$ & 0,05 & $-0,01$ & 0,00 & 0,00 \\
\hline $7 \mathrm{~A}$ & 0,06 & $-0,02$ & $-0,22$ & 0,03 & $-0,13$ & 0,11 & $-0,13$ & $-0,13$ & $-0,11$ & 0,12 & $-0,03$ & 0,03 & 0,03 & 0,06 \\
\hline $7 \mathrm{~A} \mathrm{f}$ & 0,04 & $-0,11$ & 0,11 & $-0,01$ & 0,01 & 0,02 & $-0,03$ & $-0,03$ & $-0,08$ & 0,24 & $-0,22$ & 0,10 & 0,10 & 0,12 \\
\hline
\end{tabular}

The above is consistent with the view that fourth Chakra is related to heart, cardiac plexus and vagus nerve and the first Chakra is associated with adrenals $[8,16]$. In our opinion, the significant connections between the levels of cortisol and testosterone and the parameters of the first and second Chakras, and triiodothyronine - the fifth Chakra, are quite natural.

In general, the canonical correlation between the HRV\&endocrine and virtual Chakras parameters is strong (Table 9 and Fig. 5).

\section{Table 9. Factor structure of HRV\&endocrine and virtual Chakras Roots}

\begin{tabular}{|l|c|}
\hline $\begin{array}{l}\text { Left set } \\
\text { HRV\&Hormones }\end{array}$ & R \\
\hline VLF, msec $^{2}$ & $\mathbf{0 , 4 8 9}$ \\
\hline VLF+ULF, $\mathrm{msec}^{2}$ & $\mathbf{0 , 4 7 6}$ \\
\hline Total Power HRV, $\mathrm{msec}^{2}$ & $\mathbf{0 , 4 4 9}$ \\
\hline Testosterone, Z & $\mathbf{0 , 4 2 7}$ \\
\hline HF/TP & $\mathbf{0 , 2 5 0}$ \\
\hline MxDMn HRV, msec & $\mathbf{0 , 1 3 0}$ \\
\hline Cortisol, nM/L & $\mathbf{0 , 0 7 7}$ \\
\hline Heart Rate & $\mathbf{- 0 , 3 2 0}$ \\
\hline Testosterone, nM/L & $\mathbf{- 0 , 2 8 7}$ \\
\hline LFnu, \% & $\mathbf{- 0 , 2 4 3}$ \\
\hline Amplitude Mode HRV, \% & $\mathbf{- 0 , 2 3 2}$ \\
\hline Vegetative Balance Index & $\mathbf{- 0 , 1 9 9}$ \\
\hline Baevsky's Stress Index, un & $\mathbf{- 0 , 1 9 0}$ \\
\hline
\end{tabular}




\begin{tabular}{|l|c|}
\hline Triiodothyronine, $\mathrm{nM} / \mathrm{L}$ & $-0,117$ \\
\hline Right set Chakras & $\mathbf{R}$ \\
\hline 7 E & 0,474 \\
\hline 7 E f & 0,398 \\
\hline 1 E f & 0,395 \\
\hline 4 E & 0,368 \\
\hline 2 E f & 0,364 \\
\hline 4 A f & 0,364 \\
\hline 4 E f & 0,313 \\
\hline 3 E & 0,279 \\
\hline 6 E & 0,197 \\
\hline 6 E f & 0,195 \\
\hline 4 A & 0,161 \\
\hline 2 A & 0,062 \\
\hline 5 E f & 0,056 \\
\hline 5 E & 0,052 \\
\hline 2 A f & 0,028 \\
\hline 6 A & $-0,327$ \\
\hline 3 A & $-0,327$ \\
\hline 5 A f & $-0,308$ \\
\hline 5 A & $-0,294$ \\
\hline 6 A & $-0,273$ \\
\hline 1 A f & $-0,179$ \\
\hline 7 A & $-0,069$ \\
\hline 7 A f & $-0,045$ \\
\hline
\end{tabular}

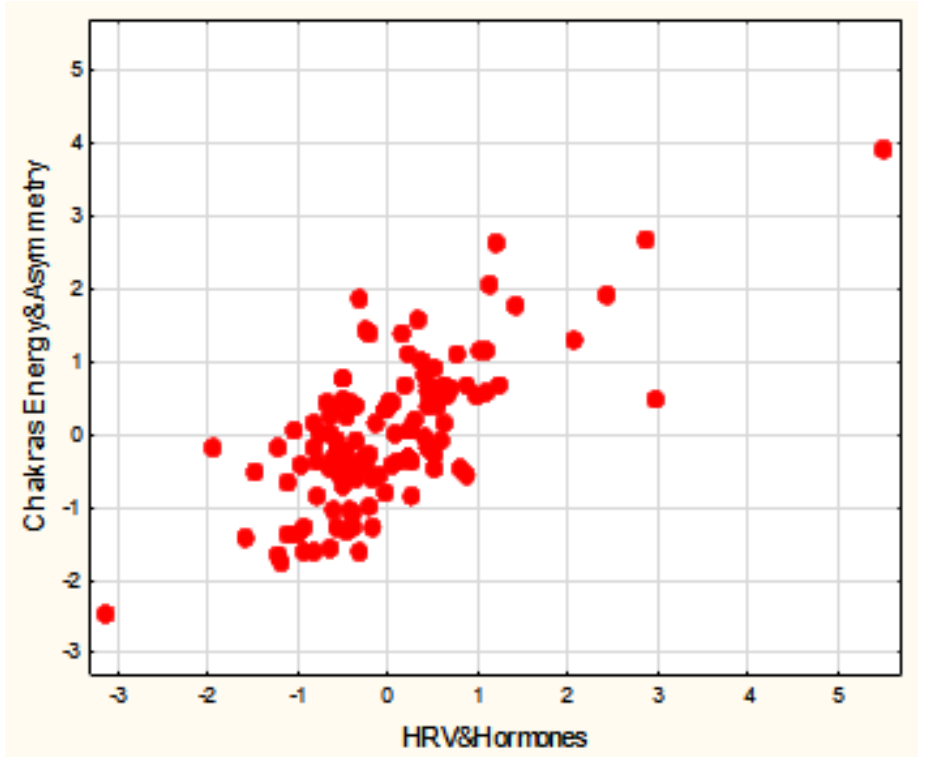

$\mathrm{R}=0,712 ; \mathrm{R}^{2}=0,507 ; \chi_{(345)}^{2}=404 ; \mathrm{p}=0,015 ; \Lambda$ Prime $=0,016$

Fig. 5. Scatterplot of canonical correlation between HRV\&Endocrine (X-line) and virtual Chakras (Y-line) parameters

In the final stage, based on the screening of the relationships between the GDI and EEG parameters, a correlation matrix was created, limited only by variables with significant coefficients (Table 10). 
Table 10. Matrix of correlations between the EEG and GDI parameters

\begin{tabular}{|c|c|c|c|c|c|c|c|c|c|c|c|c|c|c|c|c|c|}
\hline \multirow[b]{2}{*}{ Variabl } & \multicolumn{17}{|c|}{$\begin{array}{l}\text { Correlations } \\
\mathrm{N}=120\end{array}$} \\
\hline & CAK & AR & ER & ERF & CR & CRF & $\mathrm{AF}$ & SYM & Sym & $\mathrm{EF}$ & $\mathrm{CF}$ & CFF & $\mathrm{AL}$ & ALF & $E L$ & $\mathrm{CL}$ & CLF \\
\hline \begin{tabular}{|l|} 
IRA \\
\end{tabular} & $-0,10$ & 0,04 & $-0,15$ & $-0,20$ & $.0,09$ & 0,10 & $-0,03$ & 0,17 & 0,11 & $-0,05$ & $-0,06$ & 0,10 & $-0,00$ & $-0,15$ & $-0,12$ & $-0,06$ & 0,12 \\
\hline FP1H & $-0,18$ & 0,19 & 0,08 & 0,07 & 0,16 & $-0,09$ & 0,16 & 0,21 & 0,05 & 0,07 & $-0,21$ & $-0,13$ & 0,25 & 0,12 & 0,03 & $-0,23$ & $-0,13$ \\
\hline F3A & $-0,08$ & $-0,01$ & $-0,03$ & $-0,25$ & $.0,03$ & 0,10 & $-0,06$ & 0,09 & 0,07 & $-0,04$ & $-0,01$ & 0,06 & $-0,04$ & $-0,05$ & $-0,22$ & $-0,02$ & 0,08 \\
\hline F7T\% & $-0,18$ & 0,22 & 0,14 & 0,11 & 0,19 & $-0,12$ & 0,17 & 0,16 & $-0,00$ & 0,17 & $-0,22$ & $-0,13$ & 0,25 & 0,09 & 0,02 & $-0,24$ & $-0,11$ \\
\hline F8T\% & $-0,13$ & 0,21 & 0,14 & 0,03 & 0,15 & $-0,18$ & 0,17 & 0,10 & 0,08 & 0,10 & $-0,16$ & $-0,17$ & 0,25 & 0,10 & 0,12 & $-0,19$ & \\
\hline T3B\% & $-0,12$ & 0,08 & 0,26 & 0,18 & 0,06 & $-0,01$ & 0,07 & 0,07 & $-0,05$ & 0,16 & $-0,07$ & $-0,04$ & 0,09 & 0,05 & 0,15 & -0 & \\
\hline T4H & $-0,13$ & 0,22 & 0,12 & 0,07 & 0,22 & $-0,16$ & 0,19 & 0,26 & 0,13 & 0,21 & $-0,26$ & $-0,22$ & 0,27 & 0,16 & 0,01 & $-c$ & $-0,19$ \\
\hline $\mathrm{C} 3 \mathrm{~A}$ & $-0,11$ & 0,04 & $-0,03$ & $-0,25$ & $.0,07$ & 0,11 & $-0,02$ & 0,12 & 0,11 & $-0,04$ & $-0,04$ & 0,07 & 0,00 & $-0,04$ & $-0,21$ & $-0,04$ & 0,09 \\
\hline C4A & $-0,13$ & 0,07 & $-0,03$ & $-0,27$ & $.0,09$ & 0,07 & 0,01 & 0,12 & 0,10 & $-0,07$ & $-0,08$ & 0,04 & 0,04 & $-0,02$ & $-0,25$ & $-0,09$ & 0,07 \\
\hline C4D & $-0,14$ & 0,03 & $-0,06$ & $-0,09$ & $.0,04$ & 0,07 & 0,05 & $-0,02$ & $-0,25$ & 0,05 & $-0,06$ & 0,11 & 0,01 & 0,01 & 0,07 & $-0,04$ & 0,08 \\
\hline T5D $\%$ & 0,07 & $-0,11$ & $-0,28$ & $-0,05$ & 0,06 & $-0,03$ & $-0,03$ & $-0,10$ & 0,03 & $-0,19$ & 0,06 & 0,00 & $-0,07$ & 0,01 & $-0,07$ & 0,08 & 0,02 \\
\hline P3B \% & 0,01 & 0,05 & 0,27 & 0,25 & 0,02 & $-0,05$ & 0,08 & $-0,03$ & $-0,12$ & 0,16 & $-0,02$ & $-0,07$ & 0,05 & 0,13 & 0,22 & $-0,03$ & $-0,13$ \\
\hline P3A & $-0,13$ & 0,06 & $-0,04$ & $-0,23$ & $.0,07$ & 0,08 & 0,00 & 0,13 & 0,10 & $-0,06$ & $-0,05$ & 0,06 & 0,01 & $-0,06$ & $-0,26$ & $-0,04$ & 0,08 \\
\hline P3D & $-0,07$ & $-0,03$ & $-0,19$ & $-0,30$ & 0,02 & 0,05 & 0,00 & 0,04 & 0,03 & $-0,14$ & $-0,05$ & 0,07 & 0,05 & $-0,01$ & $-0,10$ & $-0,05$ & 0,06 \\
\hline P4B \% & 0,02 & 0,02 & 0,32 & 0,25 & $.0,01$ & $-0,05$ & 0,03 & $-0,04$ & $-0,13$ & 0,24 & $-0,03$ & $-0,03$ & 0,02 & 0,11 & 0,25 & $-0,01$ & $-0,05$ \\
\hline P4D & $-0,05$ & 0,01 & $-0,20$ & $-0,31$ & $.0,00$ & 0,06 & 0,04 & 0,03 & $-0,03$ & $-0,20$ & $-0,01$ & 0,06 & 0,01 & $-0,01$ & $-0,11$ & $-0,02$ & 0,02 \\
\hline $\mathrm{O} 2 \mathrm{~B} \%$ & $-0,01$ & 0,08 & 0,30 & 0,08 & 0,01 & $-0,14$ & 0,11 & $-0,00$ & $-0,07$ & 0,20 & $-0,07$ & $-0,14$ & 0,13 & 0,19 & 0,18 & $-0,09$ & $-0,23$ \\
\hline O2D & 0,12 & $-0,15$ & $-0,23$ & $-0,12$ & 0,15 & 0,12 & $-0,14$ & $-0,18$ & $-0,16$ & $-0,22$ & 0,21 & 0,15 & $-0,20$ & $-0,11$ & $-0,11$ & 0,22 & 0,18 \\
\hline
\end{tabular}

According to the canonical correlation analysis (Table 11), the factor structure of the neuro-endocrine root was headed by real testosteronemia. Next are variables with similar in sign, but much lower factor loads. Significantly, the markers of sympathetic tone are interspersed with PSD of $\boldsymbol{\delta}$ - and $\boldsymbol{\alpha}$-rhythms of EEG parameters.

Table 11. Factor structure of neuro-endocrine and GDI canonical Roots

\begin{tabular}{|c|c|}
\hline Left set & $\mathbf{R}$ \\
\hline Testosterone, nM/L & 0,557 \\
\hline LFnu, \% & 0,275 \\
\hline O2- $\delta$ PSD, $\mu V^{2} / H z$ & 0,253 \\
\hline P4- $\delta$ PSD, $\mu \mathrm{V}^{2} / \mathrm{Hz}$ & 0,242 \\
\hline LF/HF & 0,228 \\
\hline C4- $\delta$ PSD, $\mu V^{2} / H z$ & 0,225 \\
\hline T5- $\delta$ PSD, $\%$ & 0,222 \\
\hline$(\mathrm{VLF}+\mathrm{LF}) / \mathrm{HF}$ & 0,190 \\
\hline Heart Rate & 0,162 \\
\hline P3- $\alpha$ PSD, $\mu V^{2} / H z$ & 0,140 \\
\hline$(\mathrm{VLF}+\mathrm{ULF}) / \mathrm{TP}$ & 0,138 \\
\hline C3- $\alpha$ PSD, $\mu V^{2} / \mathbf{H z}$ & 0,104 \\
\hline C4- $\alpha$ PSD, $\mu V^{2} / H z$ & 0,101 \\
\hline P3- $\delta$ PSD, $\mu V^{2} / H z$ & 0,077 \\
\hline F3- $\alpha$ PSD, $\mu V^{2} / H z$ & 0,068 \\
\hline Index $\alpha, \%$ & 0,063 \\
\hline Baevsky's Stress Index, In un & 0,043 \\
\hline Testosterone, Z & $-0,399$ \\
\hline F7- $\theta$ PSD, $\%$ & $-0,381$ \\
\hline F8-0 PSD, \% & $-0,359$ \\
\hline Entropy $\mathrm{T} 4$ & $-0,306$ \\
\hline Entropy Fp1 & $-0,293$ \\
\hline P4- $\beta$ PSD, $\%$ & $-0,165$ \\
\hline
\end{tabular}




\begin{tabular}{|l|l|}
\hline T3- $\beta$ PSD, \% & $-\mathbf{0 , 1 5 6}$ \\
\hline P3- $\boldsymbol{\beta}$ PSD, \% & $-\mathbf{0 , 1 4 5}$ \\
\hline O2- $\boldsymbol{\beta}$ PSD, \% & $\mathbf{- 0 , 1 4 5}$ \\
\hline RMSSD, msec & $\mathbf{- 0 , 0 9 5}$ \\
\hline HF, msec $^{2}$ & $\mathbf{- 0 , 0 8 3}$ \\
\hline LF, msec $^{2}$ & $\mathbf{- 0 , 0 2 8}$ \\
\hline Right set & $\mathbf{R}$ \\
\hline Entropy R & $\mathbf{- 0 , 3 3 9}$ \\
\hline Entropy L & $\mathbf{- 0 , 2 3 2}$ \\
\hline Entropy L (f) & $\mathbf{- 0 , 2 9 8}$ \\
\hline Entropy F & $\mathbf{- 0 , 2 4 1}$ \\
\hline Entropy R (f) & $\mathbf{- 0 , 2 9 0}$ \\
\hline Area Left & $\mathbf{- 0 , 1 6 8}$ \\
\hline Entropy F (f) & $\mathbf{- 0 , 1 3 2}$ \\
\hline Area Frontal (f) & $\mathbf{0 , 2 4 9}$ \\
\hline Shape Coeff F (f) & $\mathbf{0 , 1 9 9}$ \\
\hline Shape Coeff L & $\mathbf{0 , 1 4 2}$ \\
\hline Shape Coeff F & $\mathbf{0 , 1 1 0}$ \\
\hline Shape Coeff R (f) & $\mathbf{0 , 1 0 6}$ \\
\hline Shape Coeff R & $\mathbf{0 , 0 8 9}$ \\
\hline Area Frontal & $\mathbf{0 , 0 5 6}$ \\
\hline Korotkov's AC & $\mathbf{0 , 0 3 7}$ \\
\hline
\end{tabular}

The constellation of variables with opposite loads is headed by testosterone, standardized by sex and age. It is accompanied by markers of vagal tone and PSD $\boldsymbol{\theta}$ - and $\boldsymbol{\beta}$-rhythm of EEG, as well as Entropy at the loci T4 and Fp1.

Interestingly, the factor structure of GDI Roots is also represented by Entropy. We have previously shown that the Entropy of GDI correlates with the entropy of both EEG and leukocytogram and immunocytogram [2]. Another constellation of GDI parameters is formed by Shape Coefficients, which give the opposite sign of the load. The last element of the factor structure is the GDI Area in the left and front projections.

Unfortunately, our possibilities for identifying cortical and subcortical regions are limited. It is traditionally believed that loci $\mathrm{C} 3 / \mathrm{C} 4$ projected hippocampus, and loci T3/T4 reflect the activity of the amygdala [17]. In practice, transcranial magnetic and direct current stimulation of the T3/T4 scalp position is used to reach the insular cortex, and F3/F4 loci - to activate the dorsolateral prefrontal cortex nuclei [review: 10]. The figures presented by Winkelmann $\mathrm{T}$ et al [20] and Yoo $\mathrm{HJ}$ et al [21] give us reason to assume that the loci $\mathrm{C} 3 / \mathrm{C} 4$ projected precentral gyrus, T3/T4 - inferior temporal gyrus, F3/F4 - caudal anterior cingulate cortex or rostral middle frontal gyrus or superior frontal gyrus, P3/P4 - supramarginal gyrus, T5/T6 - caudal anterior cingulate cortex. These cortical structures affect the activity of the vagus and sympathetic nuclei.

The canonical correlation between neuroendocrine and GDV parameters is very strong (Fig. 6). 


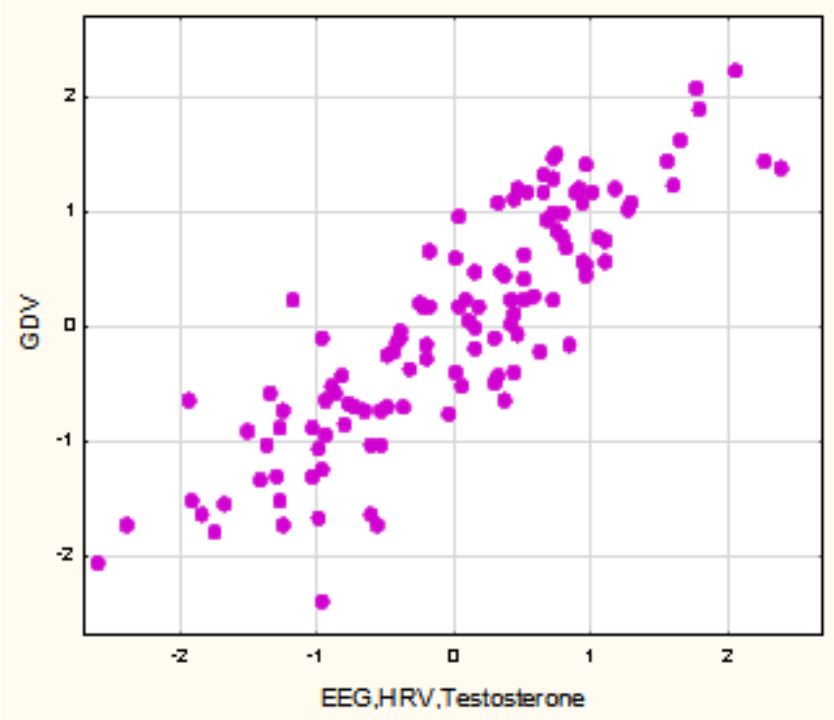

$\mathrm{R}=0,869 ; \mathrm{R}^{2}=0,755 ; \chi^{2}{ }_{(480)}=565 ; \mathrm{p}=0,005 ; \Lambda$ Prime $=0,002$

Fig. 6. Scatterplot of canonical correlation between Neuro-Endocrine (X-line) and GDV (Y-line) parameters

\section{CONCLUSION}

The above data, taken together with the previous ones, state that between parameters of neuro-endocrine-immune complex and GDV exist strong canonical correlation suggesting suitability of the latter method.

\section{ACKNOWLEDGMENT}

We express sincere gratitude to administration JSC "Truskavets'kurort" for help in carrying out EEG\&HRV registration. Special thanks to the volunteers.

\section{ACCORDANCE TO ETHICS STANDARDS}

Tests in volunteers are conducted in accordance with positions of Helsinki Declaration 1975, revised and complemented in 2002, and directive of National Committee on ethics of scientific researches. During realization of tests from all participants the informed consent is got and used all measures for providing of anonymity of participants.

\section{REFERENCES}

1. Babelyuk VE, Gozhenko AI, Dubkova GI, Babelyuk NV, Zukow W, Kovbasnyuk MM, Popovych IL. Causal relationships between the parameters of gas discharge visualization and principal neuroendocrine factors of adaptation. Journal of Physical Education and Sport. 2017; 17(2): 624-637.

2. Babelyuk VYe, Popadynets' OO, Dubkova GI, Zukow W, Muszkieta R, Gozhenko OA, Popovych IL. Entropy of gas-discharge image correlates with the entropies of EEG, immunocytogram and leukocytogram but not HRV. Pedagogy and Psychology of Sport. 2020; 6(2): 30-39.

3. Babelyuk VYe, Gozhenko AI, Dubkova GI, Zukow W, Hubyts'kyi VY, Ruzhylo SV, Fedyayeva SI, Kovalchuk HY, Popovych IL. Causal relationships between the parameters of gas discharge visualization and immunity. Pedagogy and Psychology of Sport. 2021; 7(1): 115-134.

4. Babelyuk VY, GozhenkoAI, Dubkova GI, Babelyuk NV, Zukow W, Kindzer BM, Kovbasnyuk MM, Popovych IL. Causal relationships between the parameters of gas discharge visualization and phagocytosis. Journal of Education, Health and Sport. 2021; 11(6): 268-276.

5. Babelyuk VY, Tserkovnyuk RG, Ruzhylo SV, Dubkova GI, Babelyuk NV, Zukow W, 
Popovych IL. Causal relationships between the parameters of gas discharge visualization and leukocytogram. Journal of Education, Health and Sport. 2021; 11(7): 258-269.

6. Baevskiy RM, Ivanov GG. Heart Rate Variability: theoretical aspects and possibilities of clinical application [in Russian]. Ultrazvukovaya i funktsionalnaya diagnostika. 2001; 3: 106-127.

7. Berntson GG, Bigger JT jr, Eckberg DL, Grossman P, Kaufman PG, Malik M, Nagaraja HN, Porges SW, Saul JP, Stone PH, Van der Molen MW. Heart Rate Variability: Origines, methods, and interpretive caveats. Psychophysiology. 1997; 34: 623-648.

8. Chase CR. The Geometry of Emotions: Using Chakra Acupuncture and 5-Phase Theory to Describe Personality Archetypes for Clinical Use. Med Acupunct. 2018; 30(4): 167-178.

9. Heart Rate Variability. Standards of Measurement, Physiological Interpretation, and Clinical Use. Task Force of ESC and NASPE. Circulation. 1996; 93(5): 1043-1065.

10. Iseger TA, van Bueren NER, Kenemans JL, Gevirtz R, Arns M. A frontal-vagal network theory for Major Depressive Disorder: Implications for optimizing neuromodulation techniques. Brain Stimul. 2020; 13(1): 1-9.

11. Korotkov KG. Basics GDV Bioelectrography [in Russian]. SPb. SPbGITMO(TU); 2001: 360.

12. Korotkov KG. Principles of Analysis in GDV Bioelectrography [in Russian]. SPb. Renome; 2007: 286.

13. Korotkov KG. Energy Fields Electrophotonic Analysis in Humans and Nature. Second updated edition. Translated from Russian by the author. Edited by Berney Williams and Lutz Rabe. 2014: 233.

14. Popadynets' OO, Gozhenko AI, Zukow W, Popovych IL. Relationships between the entropies of EEG, HRV, immunocytogram and leukocytogram. Journal of Education, Health and Sport. 2019; 9(5): 651-666.

15. Popovych IL, Gozhenko AI, Zukow W, Polovynko IS. Variety of Immune Responses to Chronic Stress and their Neuro-Endocrine Accompaniment. Scholars' Press. Riga; 2020: 172.

16. Puchko LG. Multidimensional Medicine. Systen of Self-diagnosis and Self-healing of Human [in Russian]. 10th ed., rev. and ext. Moskva. ANS; 2004: 432.

17. Romodanov AP (editor). Postradiation Encephalopathy. Experimental Researches and Clinical Observations [in Ukrainian and Russian]. Kyiv. USRI of Neurosurgery; 1993: 224.

18. Shaffer F, Ginsberg JP. An Overview of Heart Rate Variability Metrics and Norms. Front Public Health. 2017; 5: 258.

19. Shannon CE. Works on the theory of informatics and cybernetics [transl. from English to Russian]. Moskva. Inostrannaya literatura; 1963: 329.

20. Winkelmann T, Thayer JF, Pohlak ST, Nees F, Grimm O, Flor H. Structural brain correlates of heart rate variability in healthy young adult population. Brain Structure and Function. 2017; 222(2): 1061-1068.

21. Yoo HJ, Thayer JF, Greenig S, Lee TH, Ponzio A, Min J, Sakaki M, Nga L, Mater M, Koenig J. Brain structural concomitants of resting state heart rate variability in the young and old: evidence from two independent samples. Brain Structure and Function. 2018; 223(2): 727-737. 